\title{
The PHD Transcription Factor Cti6 is Involved in the Fungal Colonization and Aflatioxin B1 Biological Synthesis of Aspergillus Flavus
}

\section{Mengjuan Zhang}

Fujian Agriculture and Forestry University

Guanglan Lin

Fujian Agriculture and Forestry University

Xiaohua Pan

Fujian Agriculture and Forestry University

Weitao Song

Fujian Agriculture and Forestry University

\section{Can Tan}

Fujian Agriculture and Forestry University

\section{Xuan Chen}

Fujian Agriculture and Forestry University

\section{Yanling Yang}

Fujian Agriculture and Forestry University

Zhenhong Zhuang ( $\nabla$ zh_zhuang@fafu.edu.cn )

Fujian Agriculture and Forestry University https://orcid.org/0000-0003-0968-6507

\section{Research}

Keywords: Aspergillus flavus, Cti6, PHD domain, AFB1, colonization

Posted Date: August 12th, 2020

DOI: https://doi.org/10.21203/rs.3.rs-54693/v1

License: (1) (1) This work is licensed under a Creative Commons Attribution 4.0 International License. Read Full License 
1 The PHD transcription factor Cti6 is involved in the fungal colonization and

2 aflatioxin B1 biological synthesis of Aspergillus flavus

3

4 Zhang Mengjuan†, Lin Guanglan†, Pan Xiaohua, Song Weitao,Tan Can,Chen Xuan,

$5 \quad$ Yang Yanling*, Zhuang Zhenhong*

6

7 Address: Key Laboratory of Pathogenic Fungi and Mycotoxins of Fujian Province,

8 Key Laboratory of Biopesticide and Chemical Biology of Education Ministry, and

9 School of Life Sciences, Fujian Agriculture and Forestry University, Fuzhou, 350002,

10 China

$11 \dagger$ These authors contributed equally to this work.

12

13

14

15

16

17

19 18 20 21 22

*Correspondence: Zhenhong Zhuang, ZH_Zhuang@fafu.edu.cn; Yanling Yang, 1034319020@qq.com

\section{5} 6

7 9 
Abstract: Aspergillus flavus and its main secondary metabolite AFB1 pose a serious threat to several important crops worldwide. Recently, it has been reported that some PHD family transcription factors are involved in the morphogenesis and AFB1 biological synthesis in A. flavus, but the role of Cti6, a PHD domain containing protein in A. flavus, is totally unknown. The study was designed to reveal the biological function of Cti6 in the fungus by deletion of cti6, and its two domains (PHD and Atrophin-1) through homologous recombination, respectively. The results showed that Cti6 might up-regulate the mycelium growth, conidiation, sclerotia formation and AFB1 biological synthesis of A. flavus by its PHD domain, while Atrophin-1 also improved the conidiation of the fungus. The qRT-PCR analysis showed that Cti6 increased the conidiation of the fungus through AbaA and BrlA mediated conidiation pathway, triggered the formation of sclerotia by orthodox sclerotia formation pathway, and improved the production of AFB1 by orthodox AFB1 synthesis pathway. Crops models analysis showed that A. flavus Cti6 plays vital role in colonization and the production of AFB1 on the host grains mainly via PHD domain. Bioinformatics analysis showed Cti6 is conservative in Aspergillus spp., and mCherry mediated subcellular localization showed that most Cti6 accumulated in the nuclei, which reflected that Cti6 performed its important biological function in the nuclei in Aspergillus spp.. The results of the current study elucidate the roles of PHD domain containing proteins in the mechanism of the infection of crops by A. flavus, and provided a novel target for effectively controlling the contamination of Aspergillus spp. to crops. 
Keywords: Aspergillus flavus, Cti6, PHD domain, AFB1, colonization

\section{Introduction}

As a soil saprophyte worldwide, the notorious Aspergillus flavus colonizes many important crops, such as corn, peanut and cotton, and the threat of the pathogen to the life of immunosuppressed patients through aspergillosis is just second to A. fumigatus (Amaike and Keller, 2011; Tsui et al., 2011; Hedayati et al., 2007). The pathogenic fungus also causes aflatoxicosis to animal and human through the contamination of crop and feed by its most toxic secondary metabolites among known mycotoxins: aflatoxins (including aflatoxin B1, B2, G1 and G2) (Amaike and Keller, 2011; Tumukunde et al., 2020). Among aflatoxins, aflatoxin B1 (AFB1) is known to be the most toxic and carcinogenic mycotoxins known now, and it is one of the Group 1 carcinogens listed by IARC (the International Agency for Research on Cancer) (Wu et al., 2014; Xing et al., 2016). It is critical to reduce the detriment of Aspergillus flavus to crop, animal and human by manipulating the regulating mechanism of its morphogenesis, reproduction and secondary metabolism.

The morphogenesis, reproduction and secondary metabolism of A. flavus are regulated by several global transcriptional regulatory factors. As one of the global regulators, VeA is necessary for the production of mycotoxins, including AFB1, B2, cyclopianic acid and aflatrem, and sclerotia in A. flavus (Duran et al., 2007). The nuclear regulator LaeA is found to regulate the secondary metabolism in both $A$. nidulans and A. fumigatus, while in A. flavus, the global regulator negatively regulates of $\mathrm{VeA}$, and is involved in the production of conidia, sclerotia and aflatoxin (Kale et 
al., 2008). The master transcription factor A (MtfA) regulates fungal development, conidiation, sclerotia maturation, and the pathogenicity of A. flavus (Zhuang et al., 2016).

The first PHD (plant homeodomain) finger domain was found in HAT3.1 protein of Arabidopsis (Schindler et al., 1993). Several PHD finger proteins have been found in various eukaryotic species, mainly in plant and animal, while the biological function of most of them are still unidentified (Aasland et al., 1995; Wang et al., 2015). Our previous study revealed that the PHD family transcription factors are involved in the morphogenesis and aflatoxin biological synthesis in A. flavus, which showed that the PHD family transcription factor Rum1 represses conidiation, increases sclerotia formation and aflatoxin biological synthesis, up-regulates amylase activity of $A$. flavus, and involves in the colonization of the pathogenic fungus on crop kernels (Hu et al., 2018). By blast with the sequence of the main functional PHD domain of Rum1, Cti6 was found to be a PHD domain containing protein in A. flavus. In Saccharomyces cerevisiae, Cti6 was reported to be involved in the growth of yeast cells under iron-limiting condition (Puig et al., 2004). In addition, Cti6 was found to act in concert with SAGA (Spt-Ada-Gcn5-acetyltranferase) to alleviate Cyc8-Tup1 mediated repression and improve transcriptional activation in yeast (Papamichos-Chronakis et al., 2002). However the biological function of the PHD transcription factor Cti6 in the fungus has not been explored. The current study was conducted to reveal the functions of Cti6 in the virulence of A. flavus, and to find a new target for the early control of the contamination of the pathogenic fungus. 


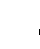

\begin{tabular}{ccc}
\hline Fungal strains & Genotype description & Reference \\
\hline A. flavus CA14 & $\Delta$ pyrG, $\Delta k u 70$ & Purchased from FGSC \\
Control (Ctrl) & $\Delta k u 70, \Delta p y r G:: p y r G$ & Used in our lab \\
$\Delta c t i 6$ & $\Delta k u 70, \Delta c t i 6:: p y r G$ & This study \\
Com-cti6 & $\Delta k u 70, \Delta c t i 6:: p y r G$, cti6::pyrG & This study \\
cti6 ${ }^{\Delta \mathrm{PHD}}$ & $\Delta k u 70, \Delta p y r G, \Delta P H D: p y r G$ & This study \\
cti6 $6^{\Delta \mathrm{ATR}}$ & $\Delta k u 70, \Delta p y r G, \Delta$ ATR:pyrG & This study \\
mcherry-cti6 & $\Delta k u 70, \Delta p y r G$, mcherry-cti6:pyrG & This study \\
\hline
\end{tabular}

100

\section{Materials and Methods}

\section{Strains and media}

A. flavus $\Delta k u 70 \Delta p y r G$ was used as the original strain in this work. All the strains used in this study are listed in Table 1. A. flavus was cultured in plates containing potato dextrose agar (PDA, 39 g/L, BDDifco, Franklin, NJ, USA), CM (6 g/L tryptone, $6 \mathrm{~g} / \mathrm{L}$ yeast extract, $10 \mathrm{~g} / \mathrm{L}$ glucose), or YES media (20 g/L yeast extract, 150 $\mathrm{g} / \mathrm{L}$ sucrose, $1 \mathrm{~g} / \mathrm{L} \mathrm{MgSO}_{4} \bullet 7 \mathrm{H}_{2} \mathrm{O}$ ). For solid media, agar was added at $15 \mathrm{~g} / \mathrm{L}$. The auxotrophic marker (pyrG-) was supplemented with uracil and uridine each at $1 \mathrm{mg} / \mathrm{mL}$ in media.

Table 1. A. flavus strains in the study. 
homologous recombination. 5' - flanking region (with primer $\mathrm{p} 1$ and $\mathrm{p} 2$, primers used in strain construction were listed in Table S1) and 3' - flanking region (primer p3 and p4) of cti6 were amplified, and fused together with A. fumigatus pyrG by nesting primer p7 and p8 according to the strategy scheme shown in Figure S1A. The fusion PCR product was transformed into the A. flavus CA14 strain by polyethylene glycol-mediated approach. Transformants were selected on a medium without Uracil and Uridine, and confirmed with PCR, RT-PCR, qRT-PCR and southern-blotting analysis. For the complementation assay, the $\operatorname{pyr} G$ in $\Delta c t i 6$ was replaced by 5'-flanking region-cti6-3'-flanking region (amplified with primer p1 and p4) under the stress of $2 \mathrm{mg} / \mathrm{mL}$ 5-FOA (5-fluorooroticacid) with the method of homologous recombination. Then, $p y r G$ gene was inserted into the transformants at the $\mathrm{N}$-terminal of cti6 gene by homologous recombination with the fusion production amplified by cti6-C-p1 and cti6-C-p4 primers as shown in the strategy scheme in Figure S1B. Finally, the constructed Com-cti6 strain was further verified using diagnostic PCR, RT-PCR and qRT-PCR. For functional domain deletion mutants, cti $^{\Delta \mathrm{PHD}}$ and cti $^{\Delta \mathrm{ATR}}$, the construction principle was the same as the construction of $\Delta c t i 6$ strain, and the constructed $c t i \sigma^{\triangle \mathrm{PHD}}$ and $c t i \sigma^{\Delta \mathrm{ATR}}$ mutant strains were confirmed by PCR and DNA sequencing in BioSune Biotechnology (Shanghai, China).

\section{Bioinformatics analysis}

The homologs of Cti6 (from A. flavus, A. oryzae, A. terreus, A. fumigatus, A. nidulans, Pyricularia oryzae, S. cerevisiae, Homo sapiens, Mus musculus and $\begin{array}{lllll}\text { Arabidopsis thaliana) were downloaded from } & \text { NCBI }\end{array}$ 
(https://pubmed.ncbi.nlm.nih.gov/), and their evolutionary relationship was analyzed with MEGA5.0. The domains of Cti6 were further identified through NCBI database (XP_002383836.1) and visualized by DOG2.0².

\section{qRT-PCR analysis}

Fungal spores $\left(10^{6} / \mathrm{mL}\right)$ were grown on PDA (for conidiation), CM medium (for sclerotia formation) or YES (for AFB1 production) for $48 \mathrm{~h}$. Thereafrer, mycelium was ground into powder with liquid nitrogen, and each $50 \mathrm{mg}$ mycelium powder was lyzed in $1 \mathrm{~mL}$ Trizol reagent (Biomarker Technologies, Beijing, China) for $5 \mathrm{~min}$. After mixing with $200 \mu \mathrm{L}$ dichloromethane and vortexed, the mixture was centrifuged under $4^{\circ} \mathrm{C}$ at $13000 \mathrm{rpm}$ for $20 \mathrm{~min}$. Then, $500 \mu \mathrm{L}$ supernatant was mixed with equal volume of isopropanol, and vortexed. This procedure was followed by centrifugation again under $4^{\circ} \mathrm{C}$ at $13000 \mathrm{rpm}$ for $18 \mathrm{~min} .400 \mu \mathrm{L}$ supernatant was transferred into a new Eppendorf tube, and mixed with $1 \mathrm{~mL} \mathrm{75 \%} \mathrm{ethanol} \mathrm{(v/v),} \mathrm{after} \mathrm{gently} \mathrm{vibrated,}$ the mixture was centrifuged under $4^{\circ} \mathrm{C}$ at $13000 \mathrm{rpm}$ for $7 \mathrm{~min}$. Finally, the supernatant was discarded, and after drying for $5 \mathrm{~min}$, the pellet (the total RNA) were dissolved with $50 \mu \mathrm{L}$ RNase free deionized water (Yang et al., 2019). The total RNA was reversely transcribed into cDNA using First Strand cDNA Synthesis Kit with oligo (dt) 18 primer (TransGen Biotech, Beijing, China). The qRT-PCR was performed following the protocol formerly described (Hu et al., 2018), and the primers used in qRT-PCR were shown in Table S2.

\section{Phenotype analysis}

For mycelium growth analysis, spores $\left(10^{3}\right)$ was point-inoculated on $15 \mathrm{~mL}$ PDA 
in petri dish at $37^{\circ} \mathrm{C}$ in dark, and the diameter of each fungal colony was measured after $5 \mathrm{~d}$. To count the conidia number, 4 cores (10 $\mathrm{mm}$ in diameter) along the radius of the colony were drilled, after which these cores were immersed in $3 \mathrm{~mL}$ water in a $6 \mathrm{~mL}$ Falcon tube. The spore suspension was transferred into a new Eppendorf tube, after the Falcon tube was vortexed, and the spores was counted under the microscope. For sclerotia formation analysis, the spores $\left(10^{3}\right)$ was point-cultured on CM medium at $37^{\circ} \mathrm{C}$ in dark for $7 \mathrm{~d}$, and the sclerotia number was counted when the fungal colony was sprayed with $70 \%$ ethanol (Hu et al., 2018).

\section{Analysis of AFB1 production}

The AFB1 production analysis was performed according to the method previously described ( Hu et al., 2018). The fungal spores $\left(10^{6} / \mathrm{mL}\right.$ ) of each A. flavus strain were inoculated into $10 \mathrm{~mL}$ of YES liquid medium, and cultured in the dark at $29{ }^{\circ} \mathrm{C}$ for $6 \mathrm{~d}$. Afterwards, AFB1 was extracted by mixing $2 \mathrm{~mL}$ YES liquid medium from the fungal culture with an equal volume of methylene chloride. The extracted AFB1 was further analyzed with TLC (thin layer chromatography) with silica gel plate. The AFB1 production of different A. flavus strain was assessed by relative quantitative analysis against the AFB1 standard sample $(0.1 \mathrm{mg} / \mathrm{mL})$.

\section{Subcellular localization}

The construction of mcherry-cti6 strain was according to previously described protocol (Liu et al., 2020). The fungal spores $\left(10^{4}\right)$ were inoculated in YES liquid medium at $37{ }^{\circ} \mathrm{C}$ for $12 \mathrm{~h}$. Collected mycelium was rinsed with PBS, then, was observed after staining with DAPI for 10 min by laser confocal scanning microscope 
(LeicaSP8).

\section{Iron concentration stress test}

In the study carried out to examine the role of Cti6 in the growth of A. flavus under iron stress condition, the liquid iron-stress medium was prepared according to a previous report on A. fumigatus with minor modifications (Reiber et al., 2005). The fungal strains Ctrl, $\Delta c t i 6, c t i 6^{\triangle \mathrm{PHD}}$, cti $6^{\mathrm{ATR}}$ and Com-cti6 were inoculated in the liquid iron-stress medium (25 g/L glucose, $3.5 \mathrm{~g} / \mathrm{L}\left(\mathrm{NH}_{4}\right)_{2} \mathrm{SO}_{4}, 2.0 \mathrm{~g} / \mathrm{L} \mathrm{KH}_{2} \mathrm{PO}_{4}, 0.5$ $\mathrm{g} / \mathrm{L} \mathrm{MgSO}_{4}$ and $8 \mathrm{mg} / \mathrm{L} \mathrm{ZnSO}_{4}, \mathrm{pH}$ 6.8), and supplied with a series concentration of $\mathrm{Fe}(\mathrm{III}) \mathrm{Cl}_{3}(0,5,10,15$ and $20 \mu \mathrm{M})$ as required. The liquid iron-stress medium with 20 $\mu \mathrm{M} \mathrm{Fe}(\mathrm{III}) \mathrm{Cl}_{3}$ was set as the control medium without iron stress. After $7 \mathrm{~d}$ culture, the wet and dry mycelia were weighed, and the relative inhibition rates of iron stress on mycelia growth were assessed (The relative inhibition rate $=$ (the weight of mycelium from the medium with $20 \mu \mathrm{M} \mathrm{Fe}(\mathrm{III}) \mathrm{Cl}_{3}$ - the weight of mycelium from each iron stress medium with from 0 to $15 \mu \mathrm{M} \mathrm{Fe}(\mathrm{III}) \mathrm{Cl}_{3}$ )/the weight of mycelium from the medium with $\left.20 \mu \mathrm{M} \mathrm{Fe}(\mathrm{III}) \mathrm{Cl}_{3}\right)$.

\section{Crop colonization assays}

The peanut and corn infection models were established based on previously described protocol (Hu et al., 2018). After aseptically treated with $8 \%$ sodium hypochlorite, peanuts and corn grains were soaked with $10^{5} / \mathrm{mL}$ fungal spores of each A. flavus strain for $30 \mathrm{~min}$. The kernels were incubated in the dark at $29{ }^{\circ} \mathrm{C}$ for 6 days, then, photographic observation was carried out. AFB1 was extracted by soaking the crop kernels in $5 \mathrm{~mL}$ dichloromethane for $20 \mathrm{~min}$. The production levels of AFB1 in 
191

192

193

194

195

196

197

198

199

200

201

202

203

204

205

206

207

208

209

210

211

212

different fungal strain treated groups were analyzed by TLC.

\section{Statistical analysis}

All data in this study were presented with the means \pm standard deviation. The analysis of statistics was implemented with the software GraphPad Prism5 (La Jolla, CA, USA), and the difference was regarded to be statistically significant when $p<$ 0.05. Error bars represented standard error for at least three replicates.

\section{Results}

\section{Cti6 is conservative in Aspergillus spp.}

The evolutionary relationship of 10 Set1 homologs (from A. flavus, A. oryzae, A. terreus, A. fumigatus, A. nidulans, Pyricularia oryzae, S. cerevisiae, Homo sapiens, Mus musculus and Arabidopsis thaliana) was analyzed with MEGA5.0. It showed that the Cti6 homologs from the fungal species were classified into one group, in which the highest similarity (100\% Identity, 100\% Query Cover) was identified between A. flavus and A. oryzae, whereas the lowest similarity (48.21\% Identity, 9\% Query Cover) was found between A. flavus and S. cerevisiae (Figure 1A). Cti6 is more conserved among Aspergillus spp., the lowest similarity is between A. flavus and A. nidulans (68.55\% Identity, $100 \%$ Query Cover) in the cladogram. The domains in Cti6 were further identified through NCBI database (Accession No.: XP_002383836.1), and visualized by DOG2.0². A PHD (plant homeodomain) and a Atrophin-1 domain were found from the Cti6 homologs of both A. flavus and A. oryzae, and only PHD domain could be found in all 10 homologous proteins (Figure 1B). 


\section{Cti6 is involved in mycelium growth and conidiation of $A$. flavus}

To evaluate the biological function of Cti6 in the growth, development and virulence of $A$. flavus, cit6 gene deletion strain $(\Delta c t i 6)$ and its complementary strain (Com-cti6) were constructed according to the strategy of homologous recombination as shown in Figure S1A and S1B. The constructed $\Delta$ cti6 and Com-cti6 A. flavus strains were further validated with diagnostic PCR, RT-PCR, qRT-PCR and southern-blotting, the results showed that both $\Delta c t i 6$ and Com-cti6 strains are successfully constructed (Figure S1C to S1F). To assess the role of the PHD and the Atrophin-1 domain inside Cti6, both domains were deleted with the method of homologous recombination Figure $\mathbf{S 2 A}$ and S2C, and the resulted PHD deletion strain $\left(c t i 6^{\triangle \mathrm{PHD}}\right)$ and Atrophin-1 deletion strain $\left(c t i 6^{\triangle \mathrm{ATR}}\right)$ was further confirmed by sequencing as shown in Figure S2B and S2D.

To explore the biological function of Cti6 and its two domains in the growth and conidiation of $A$. flavus, the constructed fungal strains were point-inoculated on PDA media for $5 \mathrm{~d}$. It showed that the mycelium became white and fluffy, and the colony became smaller than that of Ctrl strain when Cti6 was absent (Figure 2A upper panel). From the microscopic observation, few conidia could be found on the top of sporophore in the $\Delta c t i 6$ mutant (Figure 2A lower panel). To domain deletion strains, the results showed that the conidiation state and the size of the colony of $\operatorname{cti}^{\mathrm{APHD}}$ were similar to that of the $\Delta c t i 6$, but Atrophin-1 domain exhibited an insignificant effect on the mycelium growth of A. flavus (Figure 2A). The column graph established according to the size of fungal colonies reflected that Cti6 and the PHD 
domain in Cti6 played a significant role in the growth of A. flavus (Figure 2B). The conidia number was counted with hemocytometer, and the resulted column graph showed that the absence of Cti6 ( $p<0.001)$, PHD domain $(p<0.001)$ or Atrophin-1 $(p<0.01)$ domain significantly reduced the conidiation capacity of the fungus (Figure 2C). Further qRT-PCR analysis, as shown in Figure 2D, indicated that Cti6 up-regulated the conidiation of A. flavus through AbaA $(p<0.005)$ and BrlA $(p<0.001)$. The results suggested that Cti6 regulated conidiation through AbaA and BrlA mediated conidation pathway, and PHD domain played a critical role in the process.

\section{Cti6 is indispensable for the sclerotia formation in A. flavus}

To evaluate the role of Cti6 in the formation of sclerotia in A. flavus, the constructed fungal strains were point-inoculated on CM media for $7 \mathrm{~d}$. The results showed that no sclerotia was formed when Cti6 or the PHD domain inside Cti6 was missing (Figure 3A). The sclerotia number was calculated, and the resulted Figure 3B showed that without sclerotium was found in $\Delta c t i 6$ or $c t i 6^{\Delta \mathrm{PHD}}$, and the absence of Atrophin-1 domain had no effect in the formation of sclerotia compared to Ctrl. The expression levels of $n s d C, n s d D$ and $s c l R$ were further monitored by qRT-PCR, and the result showed that the deletion of cti6 gene significantly decreased the expression level of these three key sclerotia formation regulators. Above results showed that Cti6 regulated the formation of sclerotia by orthodox sclerotia formation pathway.

\section{Cti6 is critical for the biological synthesis of AFB1 in A. flavus}

In the study conducted to evaluate the biological functions of Cti6 in AFB1 


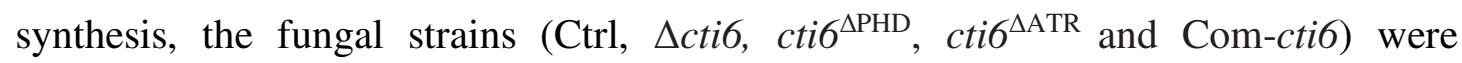
cultured with liquid YES at $29^{\circ} \mathrm{C}$ for $6 \mathrm{~d}$. Aflatoxins were extracted by dichloromethane, and analyzed by TLC. As shown in Figure $\mathbf{4 A}$ and $\mathbf{4 B}$, when Cti6 or the PHD domain inside Cti6 was absent, the production of AFB1 was dramatically decreased, On the other hand, Atrophin-1 domain appears not to participate in the process. Further qRT-PCR analysis revealed that the regulator gene aflR and aflS in the orthodox AFB1 synthesis pathway were significantly down-regulated when Cti6 was absent (Figure 4C). The aforementioned results suggested that Cti6 regulated the biological synthesis of AFB1 via its PHD domain through AflR regulated AFB1 synthesis pathway.

\section{Cti6 is involved in the colonization of $A$. flavus to crops}

A. flavus contaminates various kinds of crop kernels, especially oil plant, including peanuts and maize. To assess the role of Cti6 and its main domains in the colonization of $A$. flavus on crop grains, we inoculated the spores from Ctrl, $\Delta c t i 6$, cti $^{\triangle \mathrm{PHD}}$, $c t i 6^{\triangle \mathrm{ATR}}$ and Com-cti6 with the grains of maize and peanut according to the protocol mentioned in the Materials and Methods. The results revealed that the colonization ability of $\Delta c t i 6$ and $c t i 6^{\mathrm{AHD}}$ strain on peanut and maize grains decreased dramatically compared to Ctrl and Com-cti6 strains (Figure 5A), and the sporulation capacity of the fungi without Cti6 $(p<0.001)$ or its domain PHD $(p<0.001)$ and Atrophin-1 $(p<0.01)$ was inhibited significantly compared to the Ctrl (Figure 5B) in both crop models. The main mycotoxin of A. flavus - AFB1 was further extracted from the fungus contaminated kernels with methylene chloride, and analyzed with 
TLC. The results from TLC showed that the AFB1 mycotoxin producing capacity of both $\Delta c t i 6$ and $c t i 6^{\triangle \mathrm{PHD}}$ decreased obviously compared to the $\mathrm{Ctrl}$ and any other fungal strains in both crop grain models (Figure 5C). Further statistical analysis revealed that absence of Cti6 and PHD domain dramatically reduced the production of AFB1 $(p<0.001$, Figure 5D). Above results hinted that Cti6 of A. flavus plays critical role in colonization and AFB1 production on host crop grains mainly via PHD domain.

\section{Localization of Cti6 in the nucleus of A. flavus}

To examine the subcellular localization of Cti6 in A. flavus, a fungal strain in which Cti6 was tagged with mCherry at its $\mathrm{N}$-terminus was constructed with the strategy of homologous recombination as shown in Figure 6A. The subcelluar position of Cti6-mCherry was localized with a $552 \mathrm{~nm}$ light source, and the position of nuclei was identified with a light source of $405 \mathrm{~nm}$ wavelength. By dual-channel imaging, Cti6 was found to be accumulated in the nuclei (Figure 6B).

\section{Discussion}

The PHD transcription factor Cti6 is conservative in Aspergillus spp., and accumulated in the nuclei of $\boldsymbol{A}$. flavus.

Phylogenetic analysis showed that the Cti6 homologs from A. flavus, A. oryzae, A. terreus, A. fumigatus and A. nidulans are clustered into the same group, and the lowest similarity of Cti6 homologs among Aspergillus spp. in the cladogram is 68.55\% (between A. flavus and A. nidulans, Figure 1A). The Cti6 homologs from A. flavus and A. oryzae are identical (100\% Identity, 100\% Query Cover), and both of 
them harbor a PHD and a Atrophin-1 domain, while Atrophin-1 domain could not be found in any other Cti6 homologs among these 10 species (Figure 1B). This outcome suggested that the PHD zinc fingers domain is more conservative and more important in proper execution of the biological function of Cti6 than Atrophin-1 domain. Atrophin-1 is reported to be responsible for DRPLA (dentatorubral-pallidoluysian atrophy), a progressive neurodegenerative disorder (Wood et al., 1998). PHD domain has been reported to be involved in chromatin mediated transcriptional regulation by reading covalently modification histone sequence (Aasland et al., 1995; Sanchez and Zhou, 2011; Arrowsmith and Schapira, 2019). By co-location with mCherry, Cti6 was found to be accumulated in the nuclei of the fungus (Figure $6 \mathbf{B}$ ). The results suggested that the PHD transcriptional factor Cti6 is conservative in Aspergillus spp, and implements similar important biological functions in the nuclei of these filamentous fungi. However, there is no available report on the biological function of Cti6 in the filamentous fungi until now.

\section{Cti6 improves mycelium growth and asexual development in A. flavus.}

By point-inoculated on the PDA media or inoculated on the grains of corn and peanut, it was found that Cti6 positively improves the growth of the fungal mycelium, and up-regulates conidiation of A. flavus by transcriptional factor AbaA and BrlA (Figure2 and 5). BrlA and AbaA are two key DNA-binding transcriptional factors in regulation of asexual proliferation, in which BrlA initiates the program, and AbaA activates the development of the phialides in conidiophores (the asexual fruiting bodies) of filamentous fungi (Sewall, 1994; Mead et al., 2020). By deletion of PHD 
and Atrophin-1 domain, it was found that, similar to $\Delta c t i 6$, the fungal colony size, mycelial density and conidiation state of PHD domain deletion strain $\left(c^{2 t i} 6^{\Delta \mathrm{PHD}}\right)$ on both PDA media and crop kernels were significantly restrained compared to the Ctrl fungal strain, but Atrophin-1 domain was only involved in the sporulation of the fungus (Figure 2 and 5). The results of the study inferred that Cti6 regulates mycelium growth and asexual reproduction mainly via the assistance of PHD domain, and the PHD transcriptional factor improves the conidiation of A. flavus through AbaA and BrlA mediated sporulation-specific pathway. In view of the fact that pathogenic filamentous fungi mainly pollutes crops through their spores, the results of the current study showed Cti6 and its PHD domain are idea potential targets for reducing the colonization rate of these pathogens to various kinds of important crops.

\section{Cti6 is required in the formation of sclerotia in A. flavus.}

The analysis on the role of Cti6 in the formation of sclerotia in the study showed that Cti6 is indispensable for the formation of the structure in A. flavus (Figure 3A and 3B). As melaninized hyphal aggregates, sclerotia are commonly considered a kind of alternative reproduction form and survival structures against adverse conditions in filamentous fungi (Chang et al., 2012). It was also reported in 2009 that ascocarps, the stuctures of $A$. flavus sexual state, was embedded inside sclerotia (Horn et al., 2009). The helix-loop-helix transcriptional factor SclR, the GATA-type transcriptional factor $n s d D$ and the C2H2-Type Transcriptional Factor $n s d C$ are necessary for sexual development of filamentous fungi (Jin et al., 2011; Han et al., 2001; Kim et al., 2009). The results of this study reflected that Cti6 is required in the 
sexual reproduction of A. flavus by orthodox sclerotia regulatory transcriptional factors (NsdC, NsdD and SclR) regulated orthodox sclerotia formation pathway (Figure 3C). By the construction of PHD and Atrophin-1 domain deletion mutants, it was found that no sclerotia was formed when PHD domain was deleted, which reflected that the PHD domain plays a key role in regulating the formation of sclerotia by Cti6 (Figure 3). Therefore, Cti6 and its key domain PHD are very important breakthrough points to greatly reduce the genetic variation and survival rate of pathogenic filamentous fungi in unfavorable environmental conditions (such as in the periods of extreme cold or drought) by elimination of the structure of sclerotia.

\section{Cti6 plays a key role in biological synthesis of AFB1 in A. flavus.}

As the most toxical natural compound, AFB1, mainly produced by A. flavus, causes significant losses to agriculture. The role of Cti6 in biological synthesis of AFB1 in A. flavus was analyzed in the study. Following inoculation in liquid YES or on the kernels of corn and peanut, it was found that the absence of Cti6 greatly decreased the production of AFB1 in A. flavus by regulating the expression level of the aflatoxin biological synthesis regulatory genes aflR and aflS (Figure 4A, 4C and Figure 5C). With a GAL4-type binuclear zinc finger motif, the aflatoxin biological synthesis transcription factor AflR controls the biological synthesis of aflatoxin via regulating gene expression in the aflatoxin gene cluster (Woloshuk et al., 1994; Liu and Chu, 1998; Masanga et al., 2015). AflR interacts with aflatoxin biological synthesis regulator AflJ via Arg427, Arg 429 and Arg 431 in its C-terminal region, and the deletion of AflJ significantly decreases the expression of genes pksA, norl, verl 
and omtA in the aflatoxin biological synthesis pathway, which results in the block the synthesis of AFB1 (Chang, 2003). The deletion of Atrophin-1 domain from Cti6 obviously did not affect the production of AFB1 either in YES media or on the crop grains (Figure 4A and Figure 5C). However the absence of PHD domain significantly reduced the biological synthesis level of AFB1 both in YES liquid and on crop kernels. This outcome was similar to what was recorded in the $\Delta$ cti6 strain. These findings revealed that Cti6 plays an important role in the biological synthesis of aflatoxins mainly via its PHD domain through regulating the regulators AflR and AflJ, and that Cti6 and its PHD domain are good targets to reduce the aflatoxin contamination of crops that were polluted by A. flavus.

\section{Cti6 doesn't involve in the growth of hyphae under iron stress.}

Cti6 was reported to play a role in the growth of yeast under iron-limiting condition (Puig et al., 2004). To exam the role of Cti6 in A. flavus under iron stress, the relative inhibition rates of the fungal strains $\mathrm{Ctrl}, \Delta c t i 6, \operatorname{cti}^{\mathrm{APHD}}$, cti $\sigma^{\triangle \mathrm{ATR}}$ and Com-cti6 inoculated in series liquid iron-stress medium was examined. The results of the iron stress test by relative inhibition rates analysis showed that Cti6 isn't involved in the growth of A. flavus under iron stress conditions (data no shown). In addition, comprehensive consideration base on the aforementioned results and the results from the bioinformatics analyses reflected that the biological functions of Cti6 might be conservative just among Aspergillus spp. .

In conclusion, this study revealed that Cti6 and its PHD domain play very important roles in the morphogenesis, mycotoxin biosynthesis, and crop colonization 
of A. flavus (Figure S3). This study illuminated the potential biological functions of PHD family transcription factors in pathogenic fungi, and thereby provides idea targets to reduce the contamination rate of pathogenic filamentous fungi on crops by inhibit their sexual and asexual reproduction activities, and to reduce the mycotoxin pollution of harvested crops by restraining the biological synthesis of mycotoxins.

\section{Author Contributions:}

Z.Z. designed the experiment, wrote the manuscript, and providing financial support. Z.M. participated in all experiments and constructed domain deletion strains. L.G. constructed knockout and complementary strains. Y.Y. took part in the writing of the manuscript, and provided assistance in fungal strains construction. P.X. participated in the construction of peanut kernel infection model, and S.W. participated in the establishment of maize kernel infection model. T.C. and C.X. provided assistance in data analysis.

\section{Funding:}

This work was supported by the National Natural Science Foundation of China (No. 31772105) and the fund of Cultivation of Outstanding Youth Science and Technology Talents in Fujian Agriculture and Forestry University (xjq201410).

\section{Acknowledgments:}

We especially thank Liu Yaju and Xie Rui for their kind help in related experiments, 
411 and Afolabi Oluwatoyin Grace for her nice help in polishing the language of the

412 manuscript.

\section{Conflicts of Interest:}

415 The authors report no conflicts of interest. The authors alone are responsible for the 416 content and writing of the paper.

\section{References}

Aasland R, Gibson TJ, Stewart AF. The PHD finger: implications for

$$
\text { 1995;20(2):56-59. doi:10.1016/s0968-0004(00)88957-4 }
$$

Amaike S, Keller NP. Aspergillus flavus. Annu Rev Phytopathol. 2011;49:107-133. doi:10.1146/annurev-phyto-072910-095221

Arrowsmith $\mathrm{CH}$, Schapira M. Targeting non-bromodomain chromatin readers. Nat Struct Mol Biol. 2019;26(10):863-869. doi:10.1038/s41594-019-0290-2

Chang PK, Scharfenstein LL, Mack B, Ehrlich KC. Deletion of the Aspergillus flavus orthologue of $A$. nidulans $f l u G$ reduces conidiation and promotes production of sclerotia but does not abolish aflatoxin biosynthesis. Appl Environ Microbiol. 2012;78(21):7557-7563. doi:10.1128/AEM.01241-12

Chang PK. The Aspergillus parasiticus protein AFLJ interacts with the aflatoxin pathway-specific regulator AFLR. Mol Genet Genomics. 2003;268(6):711-719. doi:10.1007/s00438-003-0809-3 
433

434

435

436

437

438

439

440

441

442

443

444

445

446

447

448

449

450

451

452

453

454

Duran RM, Cary JW, Calvo AM. Production of cyclopiazonic acid, aflatrem, and aflatoxin by Aspergillus flavus is regulated by veA, a gene necessary for sclerotial formation. Appl Microbiol Biotechnol. 2007;73(5):1158-1168. doi:10.1007/s00253-006-0581-5

Han KH, Han KY, Yu JH, Chae KS, Jahng KY, Han DM. The $n s d D$ gene encodes a putative GATA-type transcription factor necessary for sexual development of Aspergillus nidulans. Mol Microbiol. 2001;41(2):299-309. doi:10.1046/j.1365-2958.2001.02472.x

Hedayati MT, Pasqualotto AC, Warn PA, Bowyer P, Denning DW. Aspergillus flavus: human pathogen, allergen and mycotoxin producer. Microbiology. 2007;153(Pt 6):1677-1692. doi:10.1099/mic.0.2007/007641-0

Horn BW, Moore GG, Carbone I. Sexual reproduction in Aspergillus flavus. Mycologia. 2009;101(3):423-429. doi:10.3852/09-011

Hu Y, Yang G, Zhang D, Liu Y, Lin G, Guo Z, Wang S, Zhuang Z. The PHD transcription factor Rum1 regulates morphogenesis and aflatoxin biosynthesis in Aspergillus flavus. Toxins (Basel). 2018;10(7):301. doi:10.3390/toxins10070301

Jin FJ, Takahashi T, Matsushima K, Hara S, Shinohara Y, Maruyama J, Kitamoto K, Koyama Y. SclR, a basic helix-loop-helix transcription factor, regulates hyphal morphology and promotes sclerotial formation in Aspergillus oryzae. Eukaryot Cell. 2011;10(7):945-955. doi:10.1128/EC.00013-11

Kale SP, Milde L, Trapp MK, Frisvad JC, Keller NP, Bok JW. Requirement of LaeA for secondary metabolism and sclerotial production in Aspergillus flavus. Fungal 
Kim HR, Chae KS, Han KH, Han DM. The $n s d C$ gene encoding a putative 457 C2H2-type transcription factor is a key activator of sexual development in Aspergillus

nidulans.

Genetics.

2009;182(3):771-783. doi:10.1534/genetics.109.101667

Liu BH, Chu FS. Regulation of aflR and its product, AflR, associated with aflatoxin 461 biosynthesis. Appl Environ Microbiol. 1998;64(10):3718-3723. doi:10.1128/AEM.64.10.3718-3723.1998 464 methyltransferase AflSet1 is involved in fungal morphogenesis, AFB1 biosynthesis, 465 and virulence of Aspergillus flavus. Front Microbiol. 2020;11:234. doi:10.3389/fmicb.2020.00234

Masanga JO, Matheka JM, Omer RA, Ommeh SC, Monda EO, Alakonya AE. 468 Downregulation of transcription factor aflR in Aspergillus flavus confers reduction 469 to aflatoxin accumulation in transgenic maize with alteration of host plant architecture. Plant Cell Rep. 2015;34(8):1379-1387. doi:10.1007/s00299-015-1794-9

Mead ME, Borowsky AT, Joehnk B, Steenwyk JL, Shen XX, Sil A, Rokas A. 473 Recurrent loss of $a b a A$, a master regulator of asexual development in filamentous 474 fungi, correlates with changes in genomic and morphological traits. Genome Biol 475 Evol. 2020; evaa107. doi:10.1093/gbe/evaa107 [published online ahead of print, 476 2020 May 22] 

PHD domain protein, bridges the Cyc8-Tup1 corepressor and the SAGA coactivator to overcome repression at GAL1. Mol Cell. 2002;9(6):1297-1305. doi:10.1016/s1097-2765(02)00545-2

Puig S, Lau M, Thiele DJ. Cti6 is an Rpd3-Sin3 histone deacetylase-associated protein required for growth under iron-limiting conditions in Saccharomyces cerevisiae. J Biol Chem. 2004;279(29):30298-30306. doi:10.1074/jbc.M313463200

Reiber K, Reeves EP, Neville CM, Winkler R, Gebhardt P, Kavanagh K, Doyle S. The expression of selected non-ribosomal peptide synthetases in Aspergillus fumigatus is controlled by the availability of free iron. FEMS Microbiol Lett. 2005;248(1):83-91. doi:10.1016/j.femsle.2005.05.028

Sanchez R, Zhou MM. The PHD finger: a versatile epigenome reader. Trends Biochem Sci. 2011;36(7):364-372. doi:10.1016/j.tibs.2011.03.005

Schindler U, Beckmann H, Cashmore AR. HAT3.1, a novel Arabidopsis homeodomain protein containing a conserved cysteine-rich region. Plant J. 1993;4(1):137-150. doi:10.1046/j.1365-313x.1993.04010137.x

Sewall TC. Cellular effects of misscheduled $b r l A$, $a b a A$, and wetA expression in Aspergillus nidulans. Can J Microbiol. 1994;40(12):1035-1042. doi:10.1139/m94-164

Tsui CK, Woodhall J, Chen W, Lévesque CA, Lau A, Schoen CD, Baschien C, Najafzadeh MJ, de Hoog GS. Molecular techniques for pathogen identification and fungus detection in the environment. IMA Fungus. 2011;2(2):177-189. 
501 of aflatoxins in China. World Mycotoxin Journal. 2020, 13(2):1-18. doi: 10.3920/wmj2019.2503

Wang Q, Liu J, Wang Y, Zhao Y, Jiang H, Cheng B. Systematic analysis of the maize doi:10.1038/srep25930 doi:10.1038/srep25930 

pathogenicity in Aspergillus flavus. Environ Microbiol. 2019;21(12):4792-4807. doi:10.1111/1462-2920.14825 factor mtfA governs aflatoxin production, morphological development and pathogenicity in the fungus Aspergillus flavus. Toxins (Basel). 2016;8(1):29. doi:10.3390/toxins8010029

\section{Figure legends}

Figure 1. Bioinformational analysis of Cti6. A. The construction of phylogenetic relationship among $10 \mathrm{Cti6}$ homologs (from A. flavus, A. oryzae, A. terreus, A. fumigatus, A. nidulans, P. oryzae, S. cerevisiae, H. sapiens, M. musculus and A. thaliana) with MEGA5.0.

B. The domains of Cti6 from above 10 species were identified through NCBI, and the domains were further visualized by DOG2.0².

Figure 2. Cti6 up-regulates the growth of fungal mycelium and conidiation in $A$. The“*”, “**” and “***” represents signifificant difference levels: $p<0.01, p<0.005$ and $p<0.001$, respectively. All experiments were carried out with three biological replicates, and repeated at least three times. 
543

Figure 3. Cti6 is necessary in the formation of sclerotia in A. flavus. A. The A. flavus strains were point-inoculated on $\mathrm{CM}$ media for $7 \mathrm{~d}$ at $37^{\circ} \mathrm{C}$. B. The sclerotia number was counted, and the sclerotia frmation ability of these fungal strains was compared.

C. The expression levels of transcriptional factor genes ( $n s d C, n s d D$ and $s c l R$ ) were analyzed by qRT-PCR.

Figure 4. Cti6 is critical in AFB1 synthesis in A. flavus. A. The production of AFB1 from above fungal strains was analyzed with TLC. B. The relative quantity of the amount of AFB1 according to the result from above TLC analysis. C. The expression levels of AFB1 synthesis regulator gene aflR and aflS were analyzed by qRT-PCR.

Figure 5. The role of Cti6 in the colonizaton of A. flavus on peanut and maize grains.

A. Colonization of these A. flavus strains on peanut and maize grains. B. Cti6 and its PHD domain involved in the condiation of A. flavus. C. The AFB1 production capacity of these fungal strains was analyzed with TLC analysis. D. Cti6 and its PHD domain psitively regulated AFB1 production in A. flavus.

Figure 6. The subcelluar location of Cti6. A. The construction strategy for mCherry and cti6 fusion expression A.flavus strain (mCherry-cti6). B. The subcellular location of Cti6 was showed through co-expressed mCherry. 


\section{Figures}

A

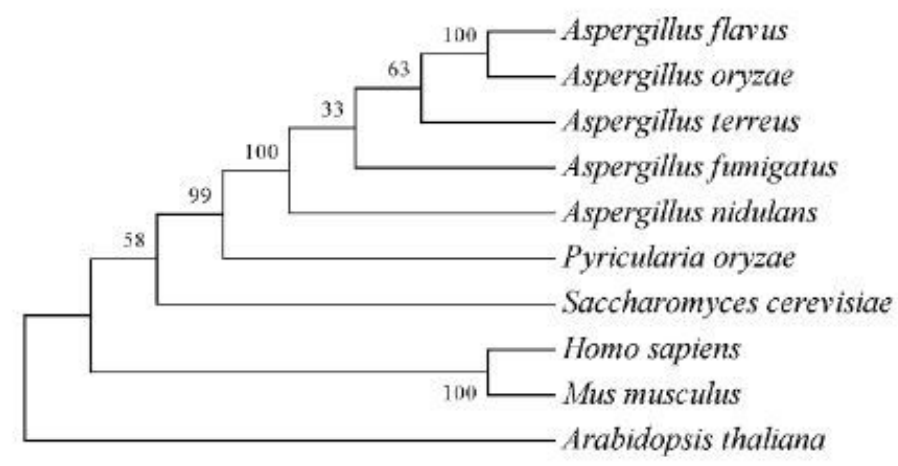

B

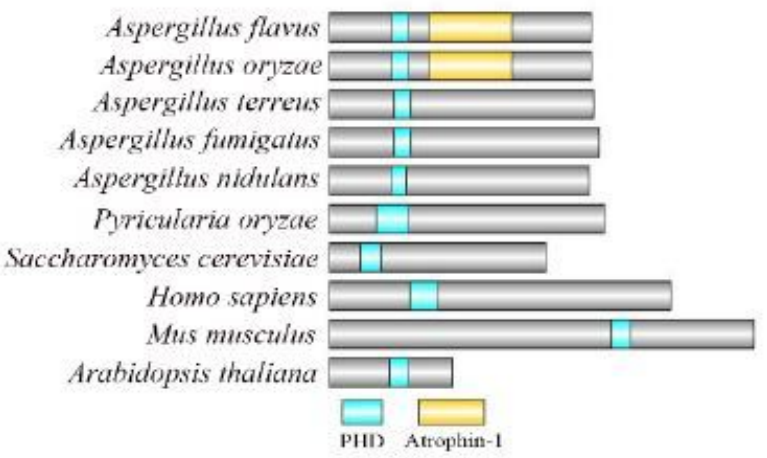

\section{Figure 1}

Bioinformational analysis of Cti6. A. The construction of phylogenetic relationship among $10 \mathrm{Cti} 6$ homologs (from A. flavus, A. oryzae, A. terreus, A. fumigatus, A. nidulans, P. oryzae, S. cerevisiae, H. sapiens, M. musculus and A. thaliana) with MEGA5.0. B. The domains of Cti6 from above 10 species were identified through $\mathrm{NCBI}$, and the domains were further visualized by DOG2.02. 
A

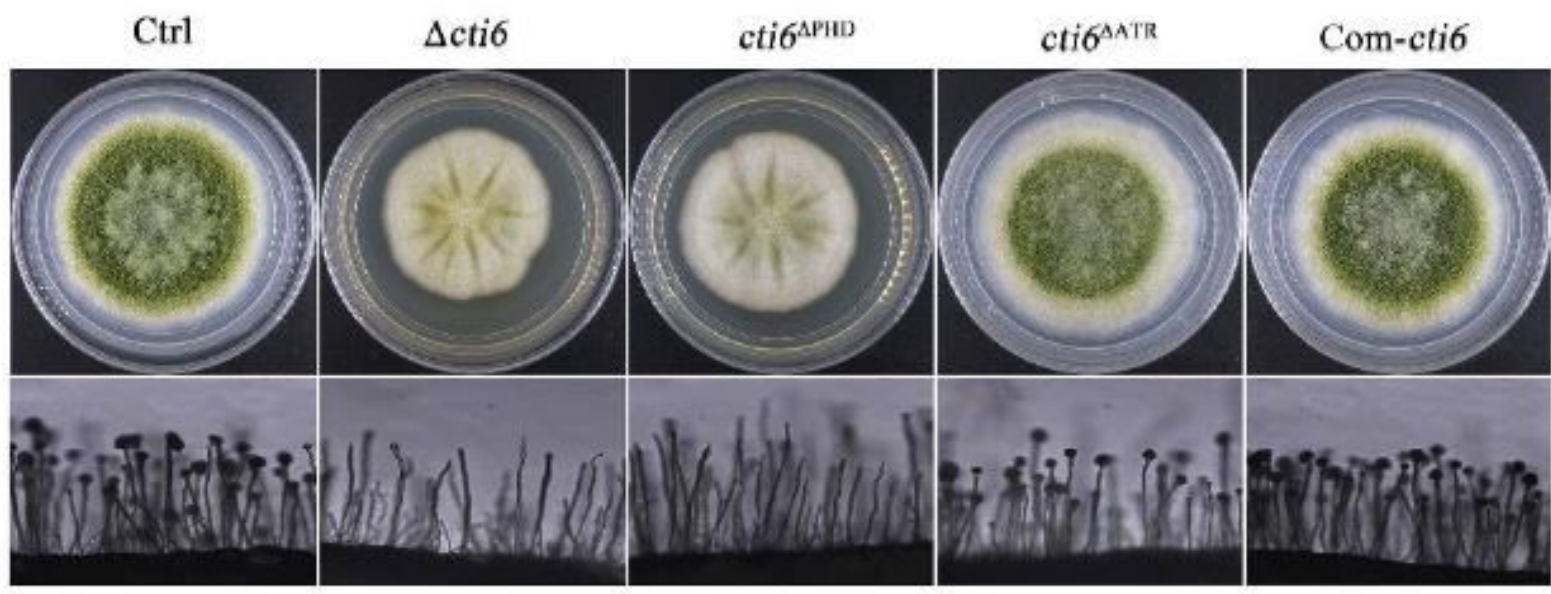

B

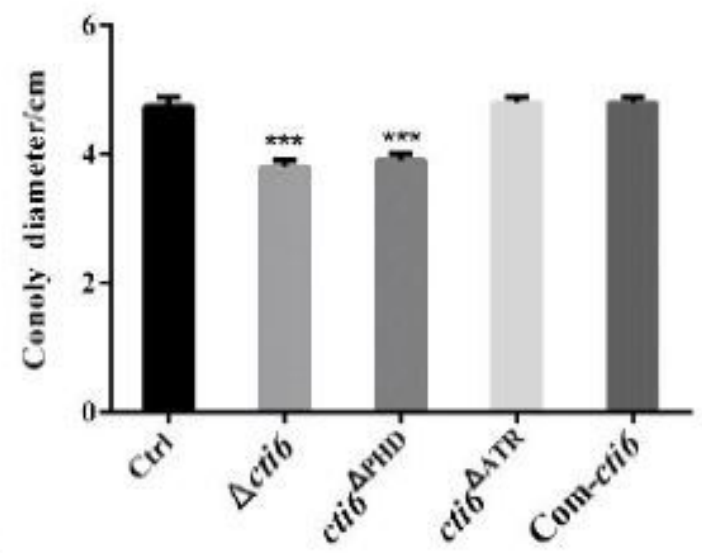

D

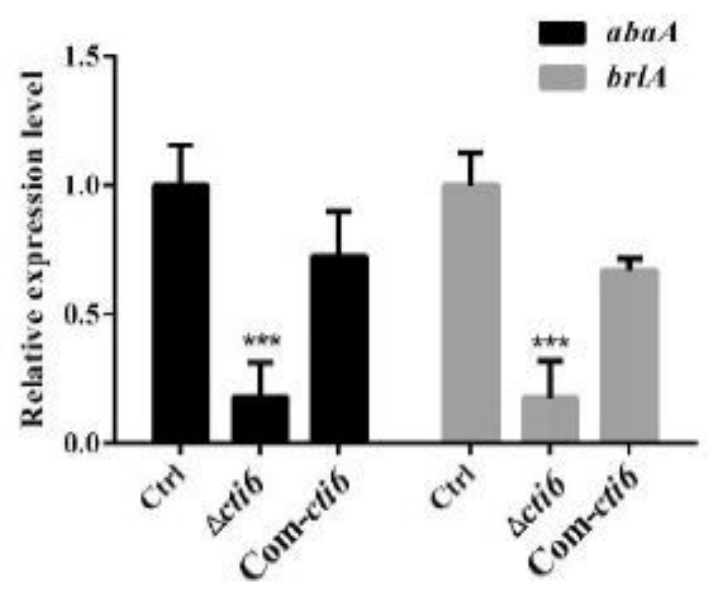

C

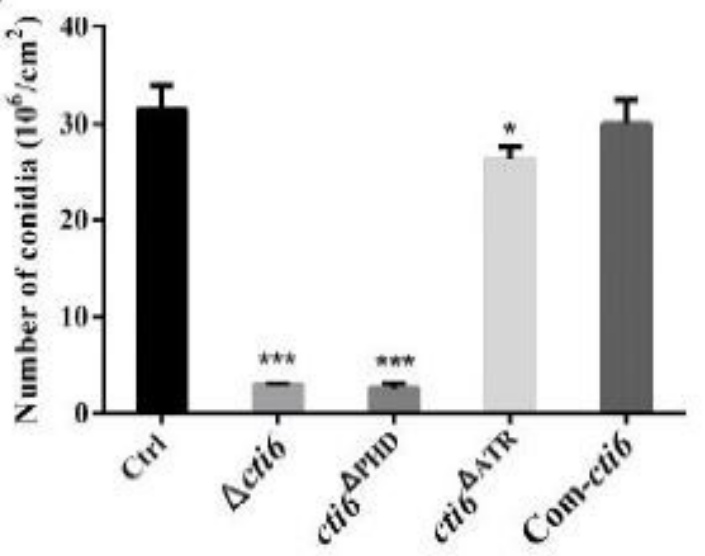

\section{Figure 2}

Cti6 up-regulates the growth of fungal mycelium and conidiation in A. flavus. A. The A. flavus strains were point-inoculated on PDA media for $5 \mathrm{~d}$ at $37 \mathrm{oC}$. B. The colony diameters were measured and represented with column graph according to the result of (A) panel. C. The number of conidia of each fungal strain on PDA medium. D. The expression level of transcriptional factor abaA and brlA genes.

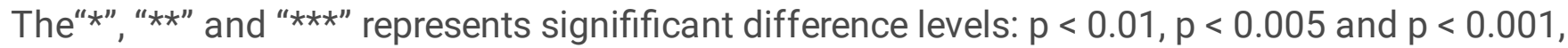


respectively. All experiments were carried out with three biological replicates, and repeated at least three times.

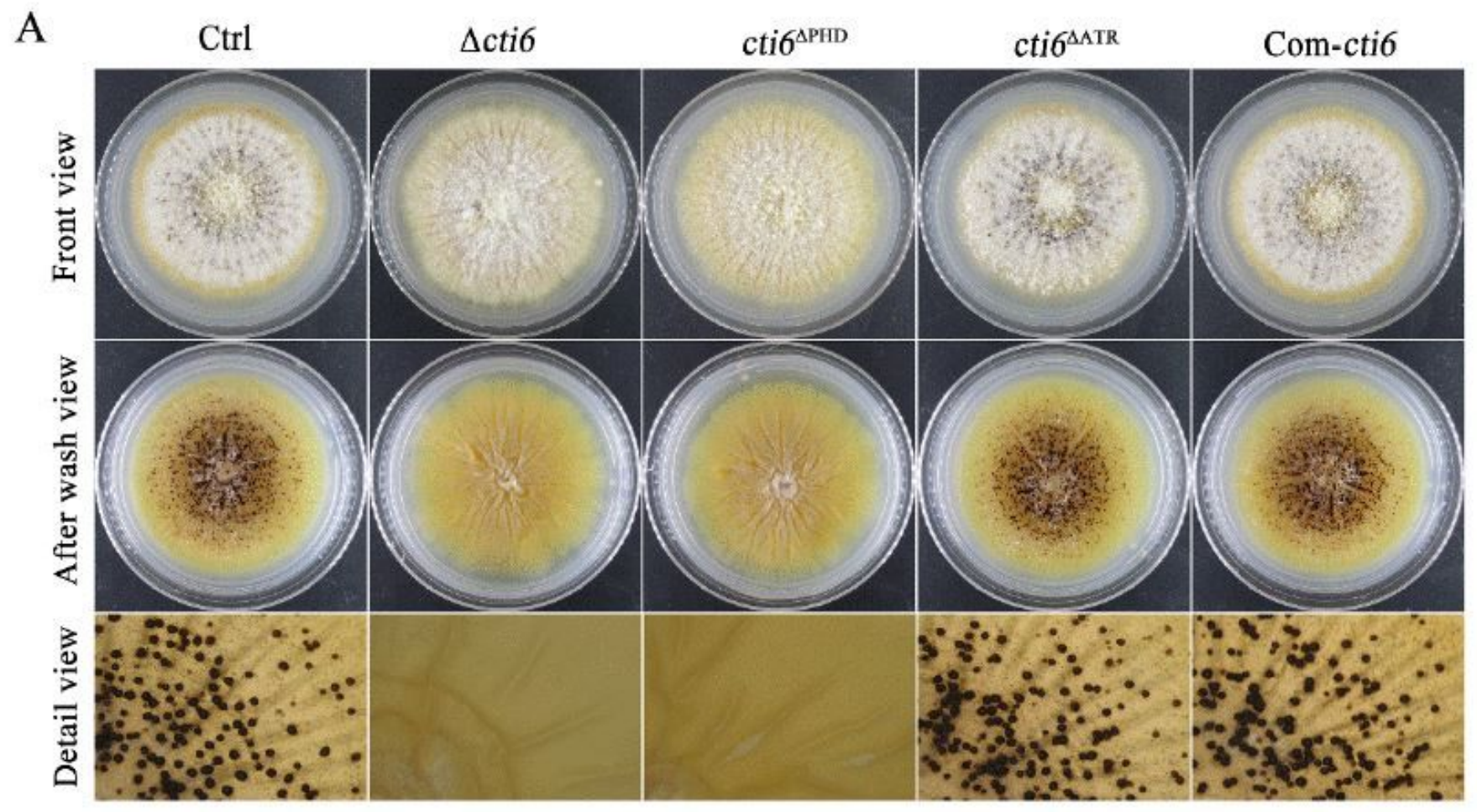

B

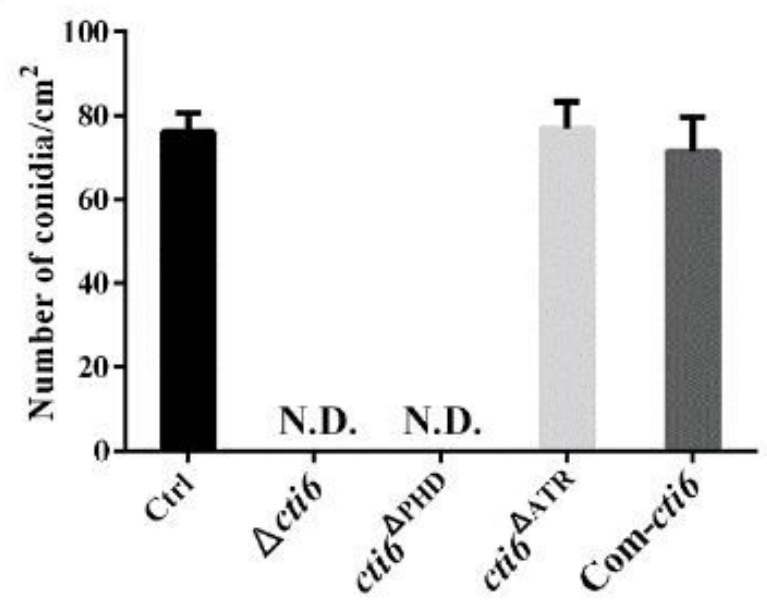

$\mathrm{C}$

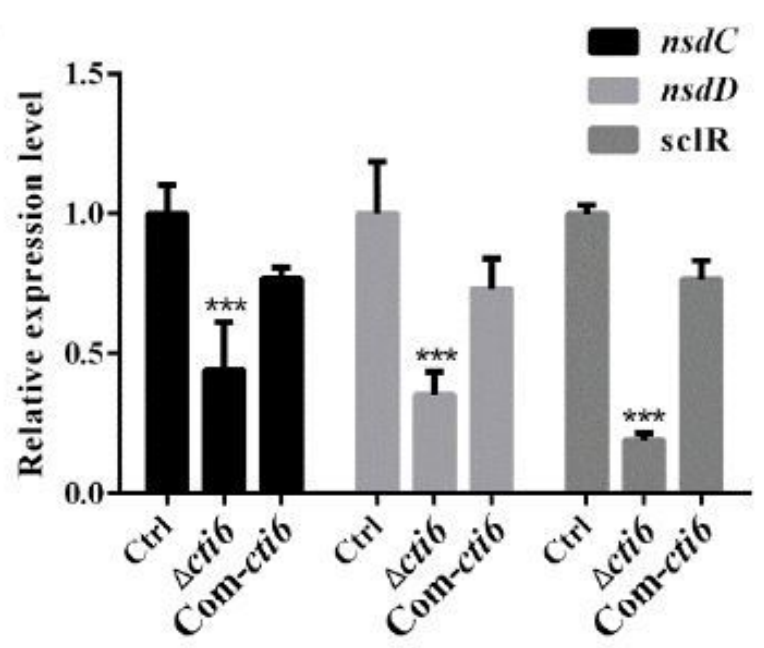

Figure 3

Cti6 is necessary in the formation of sclerotia in A. flavus. A. The A. flavus strains were point-inoculated on $\mathrm{CM}$ media for $7 \mathrm{~d}$ at $37 \mathrm{oC}$. B. The sclerotia number was counted, and the sclerotia frmation ability of these fungal strains was compared. C. The expression levels of transcriptional factor genes (nsdC, nsdD and sclR) were analyzed by qRT-PCR. 


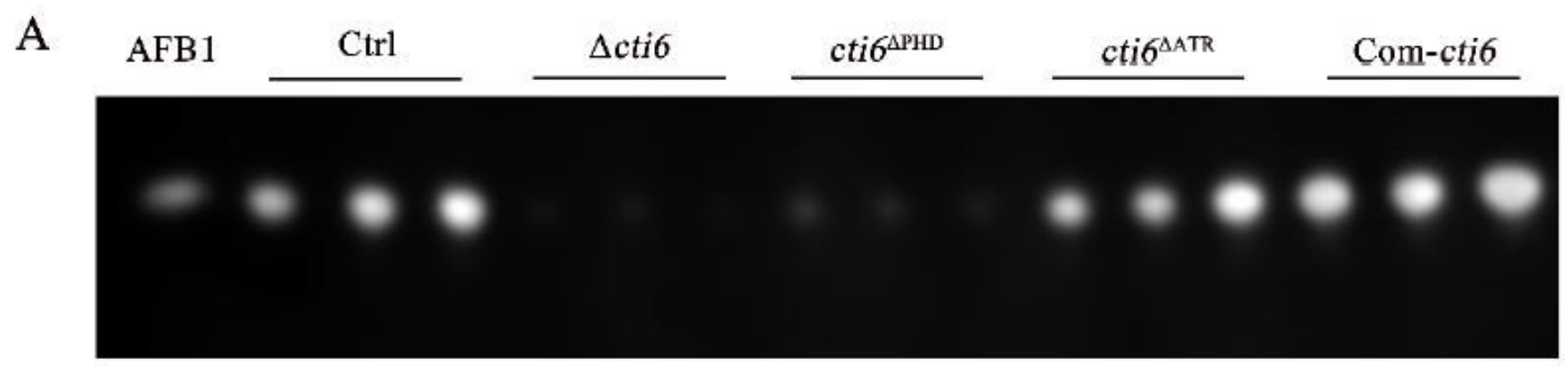

B

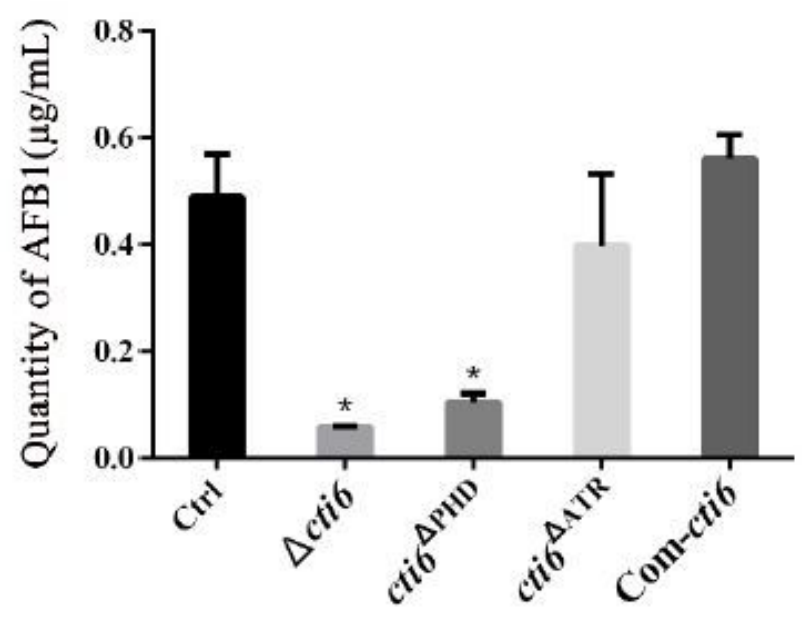

$\mathrm{C}$

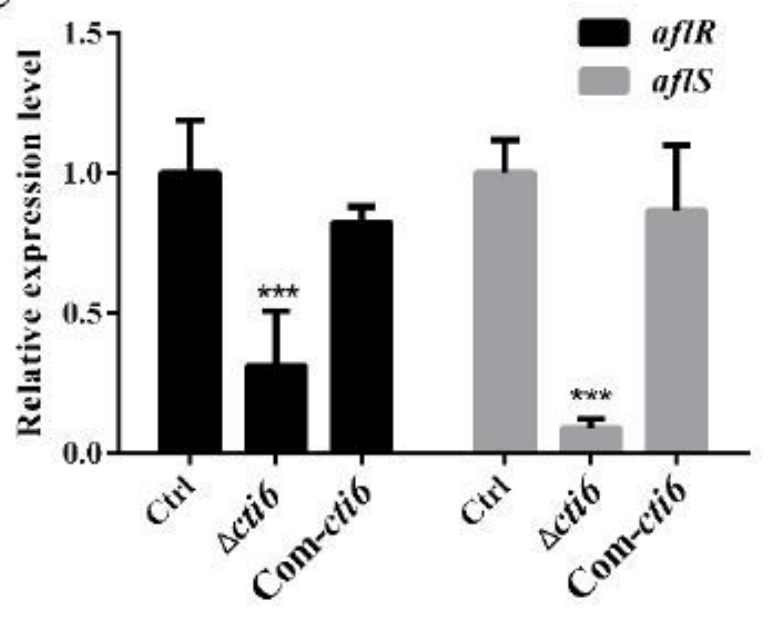

Figure 4

Cti6 is critical in AFB1 synthesis in A. flavus. A. The production of AFB1 from above fungal strains was analyzed with TLC. B. The relative quantity of the amount of AFB1 according to the result from above TLC analysis. C. The expression levels of AFB1 synthesis regulator gene aflR and aflS were analyzed by qRT-PCR. 


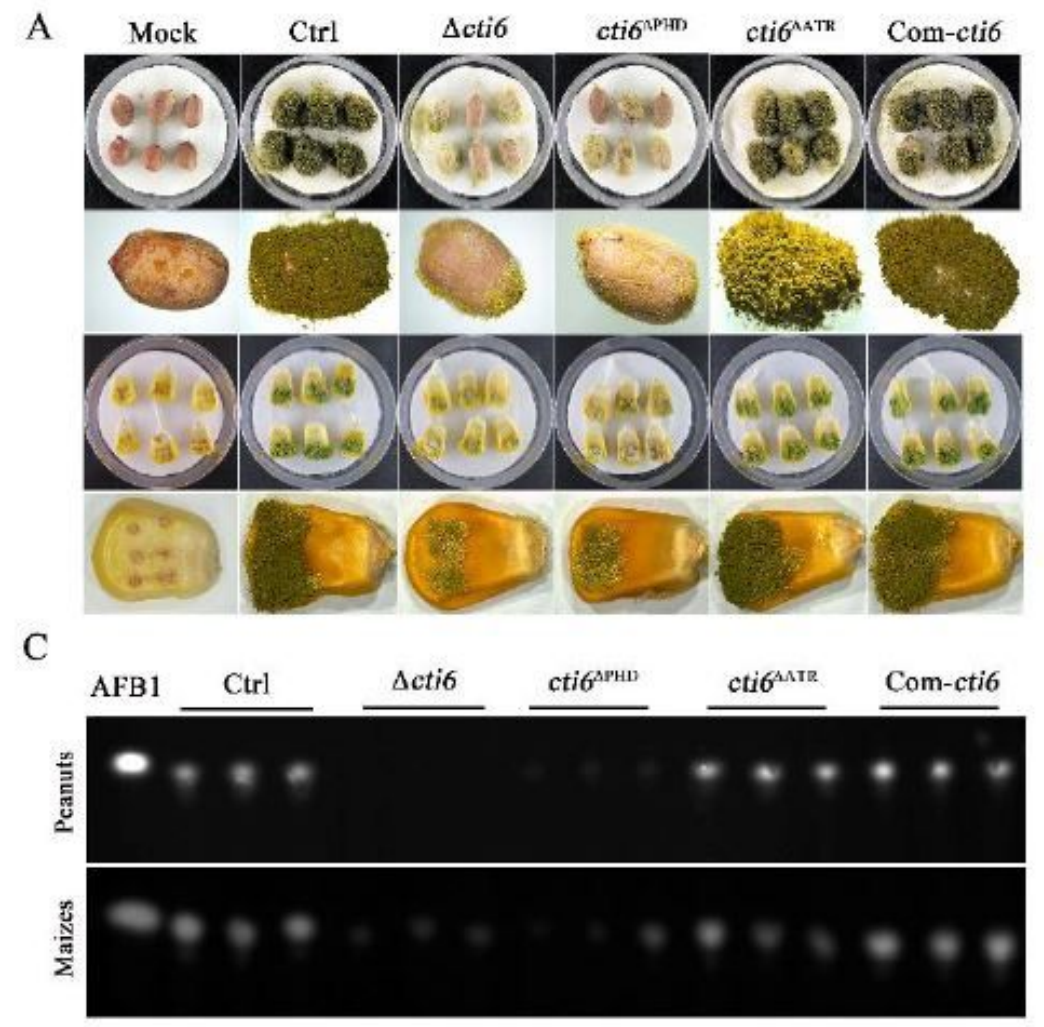

B

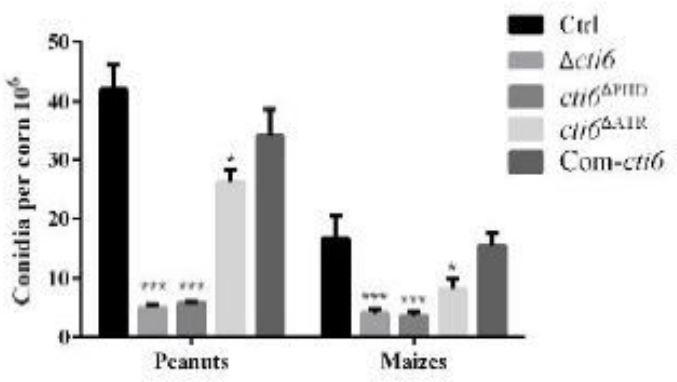

D

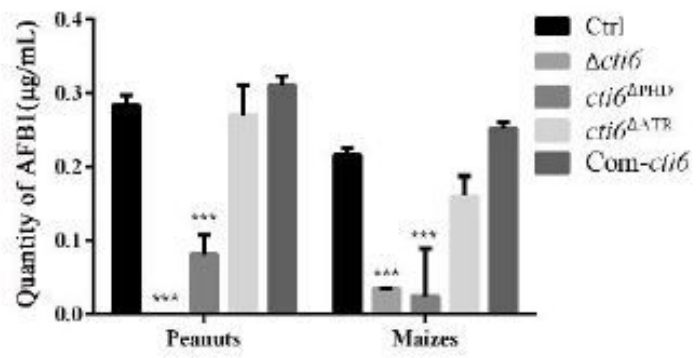

Figure 5

The role of Cti6 in the colonizaton of A. flavus on peanut and maize grains. A. Colonization of these A. flavus strains on peanut and maize grains. B. Cti6 and its PHD domain involved in the condiation of A. flavus. C. The AFB1 production capacity of these fungal strains was analyzed with TLC analysis. D. Cti6 and its PHD domain psitively regulated AFB1 production in A. flavus. 
A

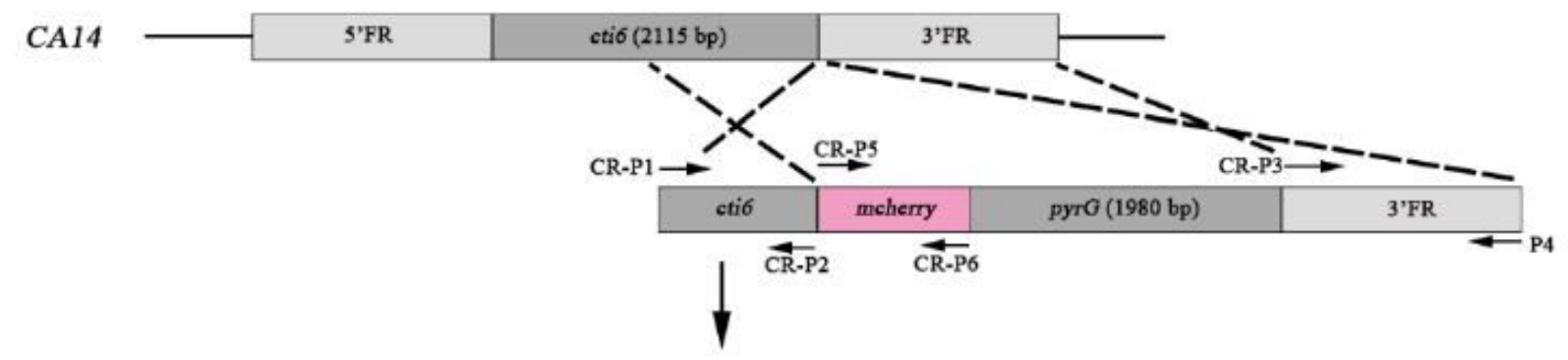

mCherry-cti6

5'FR

cti6 (2115 bp)

meherry

pyrG (1980 bp)

3'FR

B

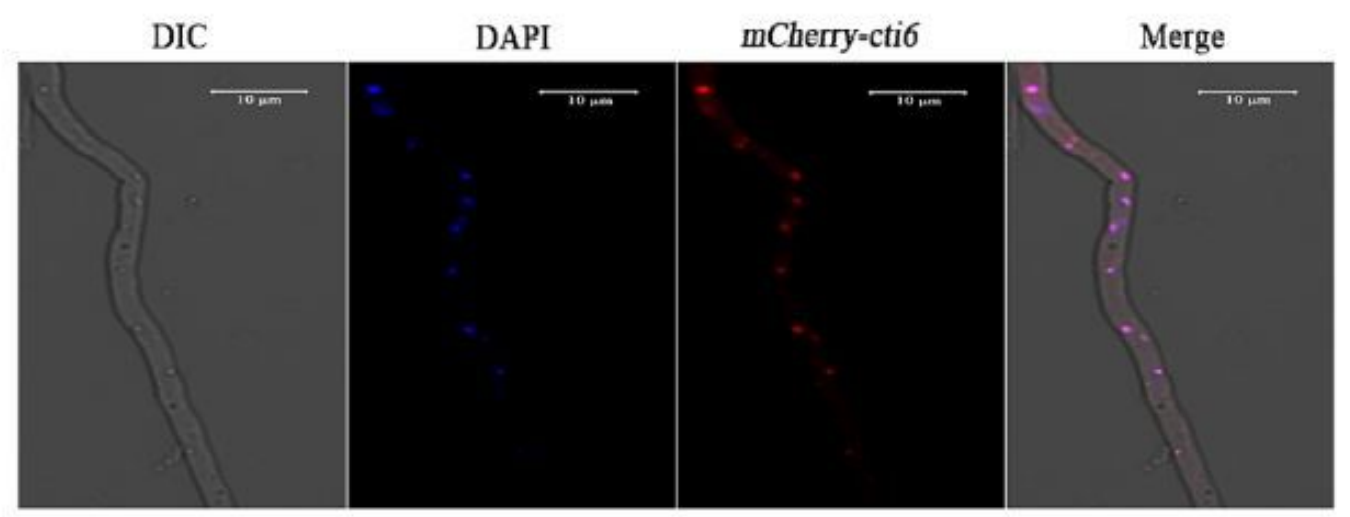

Figure 6

The subcelluar location of Cti6. A. The construction strategy for mCherry and cti6 fusion expression A.flavus strain (mCherry-cti6). B. The subcellular location of Cti6 was showed through co-expressed mCherry.

\section{Supplementary Files}

This is a list of supplementary files associated with this preprint. Click to download.

- Supplementaryfile.pdf 